\title{
Disfunción temporomandibular y depresión en adolescentes de ascendencia maya
}

\author{
Celia E. Mendiburu-Zavala ${ }^{1 *}$, América S. Castillero-Rosas ${ }^{2}$, Pedro E. Lugo-Ancona ${ }^{1}$ y \\ Josué Carrillo-Mendiburu \\ ${ }^{1}$ Facultad de Odontología, Universidad Autónoma de Yucatán, Mérida, Yucatán; ${ }^{2}$ Práctica privada, Consultorio dental, Felipe Carrillo Puerto, Quintana \\ Roo; ${ }^{3}$ Práctica privada, Consultorio de otorrinolaringólogo y cirugía de cabeza y cuello, Ciudad de México. México
}

\begin{abstract}
Resumen
Introducción: La disfunción temporomandibular (DTM) puede estar ocasionada por numerosos factores, como maloclusiones, traumatismos, bruxismo, así como por estrés y depresión. Esta última se caracteriza por un estado de profunda tristeza, abatimiento e infelicidad. Ambos trastornos pueden presentarse en la adolescencia, cuando se crean las pautas de lo que será la persona en la adultez, tanto en el aspecto psicológico como en el emocional y el anatomofisiológico. El objetivo de este estudio fue determinar la relación del grado de DTM con el grado de depresión en adolescentes de ascendencia maya de la comunidad de Yobaín, Yucatán, México. Métodos: Se llevó a cabo un estudio de tipo correlacional y transversal. Se incluyeron 210 adolescentes de ascendencia maya de entre 10 y 19 años de edad. Para el estudio se utilizaron el índice de Helkimo y la escala de depresión de Beck. Se utilizó estadística descriptiva e inferencial. Resultados: El $74.3 \%$ de los adolescentes presentó algún grado de DTM y el 51.9\%, algún grado de depresión. Se encontró que a mayor grado de DTM, mayor es el grado de depresión. La frecuencia de DTM es similar tanto para la adolescencia temprana como para la tardía, aunque en la temprana se observó mayor frecuencia de depresión grave. Conclusiones: $A$ falta de otros estudios similares, se logró sentar un precedente con este estudio acerca el estado de salud psicológica y odontológica en esta población de origen yucateco. Se estableció una pauta para acudir a otras áreas de atención médica, como psiquiatría, neurología y neuropsiquiatría.
\end{abstract}

Palabras clave: Adolescencia. Ascendencia maya. Disfunción temporomandibular. Depresión.

\section{Temporomandibular dysfunction and depression in adolescents of Mayan ancestry}

\begin{abstract}
Introduction: Temporomandibular dysfunction (TMD) is caused by multiple factors such as depression, which is characterized by a state of deep sadness, dejection and unhappiness that can be transitory or permanent. Both of these disorders can occur in adolescence, when guidelines are created for what the person will be like in adulthood for the psychological, emotional and anatomophysiological aspects. The objective of this study was to determine the relationship between the degrees of DTM and depression in adolescents of Mayan ancestry from the community of Yobain, Yucatan, Mexico. Methods: A correlational and transversal study was conducted, using the Helkimo index and the Beck depression scale, in which
\end{abstract}

Correspondencia:

${ }^{*}$ Celia E. Mendiburu-Zavala

E-mail: cel_mendi@ hotmail.com
Fecha de recepción: 04-01-2020

Fecha de aceptación: 24-02-2020

DOI: 10.24875/BMHIM.20000002
Disponible en internet: 19-05-2020 Bol Med Hosp Infant Mex. 2020;77(3):127-134

www.bmhim.com 1665-1146/C 2020 Hospital Infantil de México Federico Gómez. Publicado por Permanyer. Este es un artículo open access bajo la licencia CC BY-NC-ND (http://creativecommons.org/licenses/by-nc-nd/4.0/). 
210 adolescents aged 10 to 19 years of Mayan descent were included. Descriptive and inferential statistics were used. Results: Some degree of DTM was observed in $74.3 \%$ of the participants, and some degree of depression in $51.9 \%$. A higher degree of DTM was associated with a greater degree of depression. The frequency of DTM was similar for both categories of adolescence: early and late. However, a higher frequency of severe depression was observed in early adolescence. Conclusions: In the absence of other similar studies, a precedent was set with this study on the state of psychological and dental health in this population of Yucatan origin. Also, a guideline was established for referral to other areas of health care, such as psychiatry, neurology and neuropsychiatry.

Key words: Adolescence. Mayan ancestry. Temporomandibular dysfunction. Depression.

\section{Introducción}

La articulación temporomandibular (ATM) es la base de todo el sistema estomatognático. Al verse comprometida en su equilibrio por una disfunción en más de uno de sus componentes puede presentar alteraciones durante el tiempo de trabajo y de reposo, causando afectaciones musculares y dentales que ocasionan ruidos articulares, dolor y desviaciones mandibulares a la apertura o el cierre, y que son evidentes en más del $50 \%$ de la población ${ }^{1}$.

Un estudio realizado en la Universidad Autónoma de Yucatán, México, con 1000 pacientes de entre 15 y 85 años, reportó que el $48.8 \%$ de los individuos presentaron ruidos en la ATM² ${ }^{2}$. Esta articulación es parte fundamental del sistema estomatognático en conjunto con músculos, huesos, ligamentos y órganos dentarios, que cumplen con las funciones de masticación, deglución y habla. La ATM es una de las articulaciones más complejas, cuya función principal, la mandibulodiscal, permite la rotación del cóndilo en un eje transversal, que corresponde a los gínglimos en bisagra. Su movimiento principal, en el área temporodiscal, es el deslizamiento del cóndilo y el disco respecto al hueso temporal, lo que pertenece a sinoviales planas. Por ello, la ATM está clasificada como una articulación sinovial ginglimoartroidal ${ }^{3}$.

Se ha observado que los pacientes con dolor muscular presentan niveles más altos de somatización y depresión en comparación con los niveles más bajos de los que presentan dolor articular. Tanto la hiperactividad como los trastornos emocionales pueden aumentar el dolor, lo que provoca actividad en el sistema psicofisiológico, que también se encuentra activado por un evento nocivo, y produce una importante actividad autonómica, visceral y esquelética ${ }^{4}$. Asimismo, es posible que el desequilibrio químico en el cerebro de las personas con depresión y ansiedad genere un procesamiento anómalo de la sensación de dolor. Algunos autores han concluido que existe una relación de moderada a intensa entre la depresión y la ansiedad y los síntomas de dolor de la ATM ${ }^{4,5}$.
De acuerdo con la Organización Mundial de la Salud (OMS), la depresión afecta a más de 300 millones de personas en el mundo. De estas, aproximadamente 50 millones viven en el continente americano. Estas cifras representan un incremento del $18 \%$ entre 2005 y $2015^{6}$. En México, la depresión se presenta en el $3.3 \%$ de la población en alguna etapa de la vida. Según la Encuesta Nacional de Epidemiología Psiquiátrica (ENEP), se detectaron 96,521 nuevos casos de depresión en 2015. En ese mismo año, la encuesta realizada por el Instituto Nacional de Estadística y Geografía (INEGI) reveló que 28,447,420 mexicanos mayores de 12 años (el 29.9\% de la población) respondieron que han sentido tristeza o sensaciones que se podrían interpretar como depresión, y que el $27.5 \%$ presentó un episodio de depresión mayor antes de los 18 años de edad.

De acuerdo con el Fondo de las Naciones Unidas para la Infancia (UNICEF), la adolescencia temprana comprende de los 10 a los 14 años, y la tardía, de los 15 a los 19 años de edad ${ }^{6-9}$. Durante la adolescencia temprana se presentan los primeros cambios físicos; el más notorio es el acelerado crecimiento, seguido por el desarrollo de los órganos sexuales y las características sexuales secundarias. Durante estos procesos es probable que el individuo presente ansiedad o entusiasmo por los cambios extremos que aparecen en un tiempo muy corto ${ }^{9}$. Existen también cambios internos, menos notorios, pero igualmente intensos. El cerebro presenta un estrepitoso desarrollo fisiológico y eléctrico: el número de células cerebrales puede duplicar su número en el transcurso de un año y las redes neuronales se reorganizan, provocando consecuencias en la capacidad mental, emocional y física?.

La adolescencia es una etapa crítica para el desarrollo de la salud mental de manera positiva. Al desarrollarse adecuadamente, los individuos mantienen relaciones interpersonales que les brindan satisfacción y armonía con el entorno; pueden ser capaces de aprender y tomar decisiones correctas en su vida cotidiana. Sin embargo, la salud mental puede verse 
afectada por numerosos factores, individuales, genéticos y fisiológicos. Sin quitar relevancia a estos factores, la salud mental puede ser afectada gravemente por la adversidad social y la violencia intrafamiliar, escolar y en sus comunidades ${ }^{9}$.

Valorando lo anterior, existe un alarmante problema de depresión al alza en el Estado de Yucatán: 246 personas se suicidaron durante el año 2018. La edad fue un factor de impacto: en 2017 se registraron tres suicidios en menores, mientras que esta cifra se elevó a más del doble (cinco de sexo masculino y dos de sexo femenino) en 2018. Silveira Sáenz indicó a la Jornada Maya que los jóvenes son el grueso de la población, por lo que, probablemente, se han registrado más casos de suicidios en personas de 15 a 18 años en los últimos años. En el mismo reportaje, Tomás Dzul Peniche, psicoterapeuta humanista, reveló que «a veces piensan que los jóvenes o niños están jugando, hasta que ejecutan el acto. Si no fallece el menor, consiguen ayuda, pero en ocasiones apenas superan el cuadro de depresión y observan mejoras físicas, conductuales o emocionales, dejan de traerlos al tratamiento y eso puede resultar contraproducente $\$$.

Según la Unidad de Salud Mental de la Secretaría de Salud Estatal de Yucatán, la depresión es el principal detonador para que las personas atenten contra su vida, por lo que se ha convertido en un problema de salud pública, ya que ocurre un suicidio cada 33 horas. En promedio, la tasa de suicidio en el país es de 4.5 por cada 100,000 habitantes, mientras que en Yucatán es de 10.5 por cada 100,000 habitantes $^{11}$. El psiquiatra César Iván Espadas Sosa, expresidente de la Academia Yucateca de Medicina y Cirugía, A.C., coincide con estos resultados y enuncia que, aunque pudieran estar presentes el alcohol o alguna otra droga, la depresión es un factor fundamental vinculado con la mayoría de los suicidios ${ }^{12}$.

Con base en el registro de la encuesta intercensal realizada por el INEGI en 2016, hay 2227 habitantes en la comunidad de Yobaín, Yucatán, México, de los cuales 1114 son de sexo masculino y 1113 son de sexo femenino, la mayoría de ascendencia maya. Dentro de esta población se cuentan 420 adolescentes: 216 de sexo masculino y 204 de sexo femenino ${ }^{8}$. Por lo tanto, el objetivo de la presente investigación es determinar la relación del grado de disfunción temporomandibular (DTM) con el grado de depresión en adolescentes de ascendencia maya de la comunidad de Yobaín, Yucatán, durante el periodo de marzo a mayo de 2018.

\section{Métodos}

Se realizó un estudio de tipo correlacional de corte transversal. Las variables estudiadas fueron el grado de depresión, evaluado con la Escala de Beck; la DTM, determinada con el Índice de Helkimo; la adolescencia temprana (10-14 años) o tardía (15-19 años), según UNICEF; la ascendencia maya (adolescentes, padres y abuelos con ambos apellidos de ascendencia maya); y el sexo (masculino o femenino), según la OMS. El universo de la prueba se conformó por 380 adolescentes de 10-19 años de la comunidad de Yobaín, Yucatán, de marzo a mayo de 2018. El tamaño de la muestra fue de 210 adolescentes de ascendencia maya que nacieron en la comunidad de Yobaín y habitaban en el mismo lugar. Los criterios de inclusión fueron los siguientes: adolescentes con ambos apellidos de origen maya, de 10 a 19 años de edad, de ambos sexos, que aceptaran participar en el estudio y contaran con la autorización de sus padres o tutores en caso de ser menores de edad (10-17 años). Se excluyeron del estudio aquellos con antecedentes de algún traumatismo en la cabeza o el cuello, o que estuvieran bajo los efectos del alcohol, drogas u otras adicciones que pudieran interferir con el estudio. Se descartaron los que no contestaron correctamente la encuesta. El tipo de muestreo fue no probabilístico por conveniencia.

Se realizaron visitas domiciliarias para recopilar los datos. Para ordenarlos, la comunidad se dividió en cuatro grupos: el primero comprendió del 15 al 20 de marzo; el segundo, del 21 al 26 de marzo; el tercero, del 26 al 30 de marzo; y el cuarto, del 1 al 10 de abril de 2018.

En cada domicilio se encuestó a los participantes acerca de los apellidos de sus padres, sus abuelos y los propios, para confirmar si cumplían con el indicador de ascendencia maya. Se les explicó en qué consistía la investigación. Si eran menores de edad (10-17 años), el padre o tutor estuvo presente para escuchar la explicación. Si el adolescente decidía participar, el padre o tutor firmaba la carta de consentimiento informado y voluntario. Se les proporcionó un lápiz para que los participantes respondieran el índice de Helkimo, modificado por Rani, et al. ${ }^{13}$ y validado por Da-Cunha, et al. ${ }^{14}$, para evaluar el grado de DTM, los signos y los síntomas. Se siguieron los criterios de la Norma Oficial Mexicana NOM-013-SSA2-2015 ${ }^{15}$ para la prevención y el control de enfermedades bucales, aplicando las medidas pertinentes de barrera de protección.

El investigador se colocó de frente al paciente y llevó a cabo las mediciones indicadas con un Vernier 
estándar, abatelenguas y estetoscopio. Se establecieron los siguientes puntos de estudio y criterios:

- Limitación en el rango del movimiento mandibular.

La apertura bucal máxima se determinó colocando

el Vernier desde el borde incisal superior hasta el borde incisal inferior en la línea media, sin forzar la apertura bucal del adolescente. Se calificó de la siguiente manera: > $40 \mathrm{~mm}$, sin limitación o apertura normal (0 puntos); 30-39 mm, limitación leve (1 punto); $<30 \mathrm{~mm}$, limitación importante (5 puntos).

- Máximo deslizamiento a la derecha y a la izquierda. Se consideró la medición a partir del deslizamiento que efectúa la mandíbula hacia la derecha y la izquierda desde la posición de máxima intercuspidación. Se tomó la línea interincisiva como punto de referencia (cuando esta coincidió) o la línea incisiva superior (en caso de desviaciones de la línea media): $>7 \mathrm{~mm}$, deslizamiento normal (0 puntos); 4-6 mm, limitación leve del deslizamiento (1 punto); 0-3 mm, limitación importante del deslizamiento (5 puntos).

- Máxima protrusión. Se pidió al paciente que realizara dicho movimiento llevando la mandíbula hacia adelante y, utilizando el Vernier, se midió el avance desde el borde incisal superior hasta el borde incisal inferior en la línea media: $>7 \mathrm{~mm}$, movimiento protrusivo normal (0 puntos); 4-6 mm, limitación leve del movimiento protrusivo (1 punto); 0-3 mm, limitación importante del movimiento protrusivo (5 puntos).

- Índice de movimiento. Se realizó la suma de la puntuación obtenida en cada ítem anterior. Según el rango de movimiento efectuado por el paciente, se consideró como movilidad normal (0 puntos), moderado deterioro de la movilidad (1-4 puntos) o grave deterioro de la movilidad (5-20 puntos).

También se revisaron las alteraciones de la función articular mediante palpación digital, auscultación y observación. Se indicó abrir y cerrar la boca en apertura máxima al adolescente y se comprobó si existía sonido articular unilateral o bilateral, así como la desviación mandibular en ambos movimientos. Se incluyó la presencia de traba o luxación mandibular, con o sin sonido, mediante la palpación de la región articular durante los movimientos de apertura y cierre. Se consideraron las siguientes características:

- Ruido articular: crepitación o chasquido. Se auscultaron con ayuda del estetoscopio o por simple audición.

- Traba: bloqueo ocasional de corta duración.

- Luxación: dislocación del cóndilo con fijación fuera de la cavidad.

Los valores se ponderaron y se registraron de la siguiente manera: apertura y cierre mandibular sin desviaciones ni sonido (0 puntos), desviación mandibular o presencia de ruidos articulares durante el movimiento de apertura 0 ambas (1 punto), o traba 0 bloqueo de corta duración con 0 sin sonido (5 puntos).

Por otro lado, se evaluó el dolor durante el movimiento mediante las referencias dadas por el adolescente durante la exploración clínica, con los siguientes criterios: movimiento mandibular sin dolor (0 puntos), dolor referido a un solo movimiento (1 punto) o dolor referido a dos o más movimientos (5 puntos).

Asimismo, se evaluó el dolor muscular. Mediante la palpación con la superficie palmar de los dedos, se aplicó una presión a lo largo del músculo igual a la presión aplicada en la mano entre los dedos pulgar e índice. La presión debió ser suave, pero mantenida (por 1-2 segundos), con un ligero movimiento circular. Con el individuo en posición de reposo, se procedió a palpar los músculos masticatorios de la siguiente forma: se palparon las fibras anteriores, medias y posteriores del músculo temporal de forma bimanual, utilizando para ello los dedos índice, medio, anular y meñique de ambas manos. La palpación del músculo masetero también se realizó con las dos manos. Al ser un músculo elevador, el músculo pterigoideo medial o interno se contrae cuando se juntan los dientes, por lo que la manipulación funcional se realizó pidiendo al paciente apretar los dientes con fuerza. Este músculo se distiende al abrir ampliamente la boca, por lo que, en caso de dolor, la apertura amplia lo incrementaría.

Debido a que el pterigoideo lateral inferior se contrae al abrir la boca o al realizar el movimiento protrusivo, la manipulación más eficaz consistió en que el paciente llevara a cabo una protrusión en contra de una resistencia realizada por el examinador. El pterigoideo lateral superior se contrae con los músculos elevadores, por lo que se pidió al paciente apretar con fuerza los dientes. Se determinó la sensibilidad si el paciente refirió dolor a la palpación en algunas zonas de estos músculos. La puntuación se asignó de acuerdo con los siguientes criterios: sin dolor de los músculos masticatorios en actividad (0 puntos), dolor al menos en tres de los músculos masticatorios en actividad (1 punto), o dolor en cuatro o más de los músculos masticatorios en actividad (5 puntos).

Se evaluó el dolor en la ATM mediante el examen clínico, por lo referido por el paciente o a través de ambos durante el interrogatorio. Se colocaron los dedos índice en la zona preauricular, se ejerció presión bimanual y se comprobó la presencia de dolor a la palpación. Posteriormente, la presión se realizó con los mismos dedos introducidos en los conductos auditivos 
externos. Los criterios de valoración fueron los siguientes: sin dolor espontáneo ni a la palpación (0 puntos), dolor a la palpación en la región preauricular (1 punto), o dolor a la palpación en la región preauricular y en los conductos auditivos externos (5 puntos).

Finalmente, se sumaron los valores asignados de los cinco ítems del examen clínico. Se alcanzó un máximo de 25 puntos, a partir del cual se realizó la clasificación del grado de DTM: 0 puntos, sin DTM; 1-9 puntos, leve; 10-19 puntos, moderada; 20-25 puntos, grave.

A continuación se determinó el grado de depresión mediante la aplicación del Cuestionario de Depresión de $B{ }^{16}{ }^{16}$. En este cuestionario aparecen varios grupos de afirmaciones que los adolescentes leyeron con atención y señalaron cuáles de las afirmaciones de cada grupo describían mejor cómo se habían sentido durante esa última semana, incluyendo el día de la aplicación. Si dentro de un mismo grupo había más de una afirmación que el adolescente consideró aplicable a su caso, la seleccionó también. Cada afirmación se calificó de 0 a 3.

Para entender el grado de depresión se ponderó con los siguientes intervalos: 1-10, altibajos considerados normales; 11-16, leve perturbación del estado de ánimo; 17-20, estados de depresión intermitentes; 21-30, depresión moderada; 31-40, depresión grave; más de 40, depresión extrema. Al obtener una puntuación persistente de 17 o más se informó al adolescente 0 al tutor - de manera sutil, con tacto, respeto y dignidadde que necesitaría ayuda profesional, y se sugirió acudir a un especialista o a la clínica para ser reevaluado y dar seguimiento profesional a su caso.

\section{Análisis estadístico}

Se utilizaron estadísticos descriptivos (frecuencias y porcentajes) para caracterizar las variables en aquellos objetivos que requirieron prevalencias. Para las comparaciones se utilizó la prueba no paramétrica $U$ de MannWhitney, debido a que se busca comparar una variable ordinal (DTM/depresión) en dos grupos (por sexo y por edad). Para analizar la relación entre el grado de DTM y la depresión se utilizó la correlación no paramétrica de Spearman. Se consideró un nivel de confianza del $95 \%$, es decir, se consideraron resultados estadísticamente significativos los que tuvieron un valor de $p<0.05^{17}$.

\section{Resultados}

En este estudio se evaluó una muestra de 210 adolescentes de ascendencia maya: 116 de sexo femenino



Figura 1. Porcentaje de los grados de depresión según el Cuestionario de Beck por clasificación de las categorías de edad en adolescentes de ascendencia maya en Yobaín, Yucatán, México.

y 94 de sexo masculino, con un promedio de edad de $13.91 \pm 2.78$ años.

El grado de DTM observado fue leve en el $74.3 \%$ $(n=156)$, moderado en el $19.5 \%(n=41)$, grave en el $1.9 \%(n=4)$ y sin DTM en el $4.3 \%(n=9)$.

Se analizó si existían diferencias en el grado de DTM según el grupo de edad a partir de una comparación de grupos. No se detectaron diferencias estadísticamente significativas $(U=4829 ; p=0.104)$; es decir, las prevalencias de DTM fueron similares en ambos grupos de edad.

Se determinó la DTM según el sexo y se detectaron diferencias estadísticamente significativas $(U=4447$; $p=0.003$ ). Las prevalencias fueron diferentes entre sexos: el sexo femenino presentó con mayor frecuencia DTM en grado moderado a grave.

Por otro lado, se realizaron estadísticos descriptivos (frecuencias y porcentajes) según el grado de depresión obtenido a partir del Cuestionario de Depresión de Beck. Se observó un grado de depresión considerado normal en el $48.1 \%$ ( $n=101)$, leve en el $22.9 \%(n=48)$ leve, intermitente en el $6.7 \%(n=14)$, moderado en el $15.2 \%(n=32)$ y depresión extrema en el $7.1 \%(n=15)$.

Para determinar la depresión según la edad, nuevamente se dividió la muestra según las categorías de adolescencia temprana y tardía. Ambos grupos mostraron prevalencias similares: el grado de depresión más frecuente fue normal, seguido de depresión leve (Figura 1). Sin embargo, los adolescentes tardíos presentaron un 


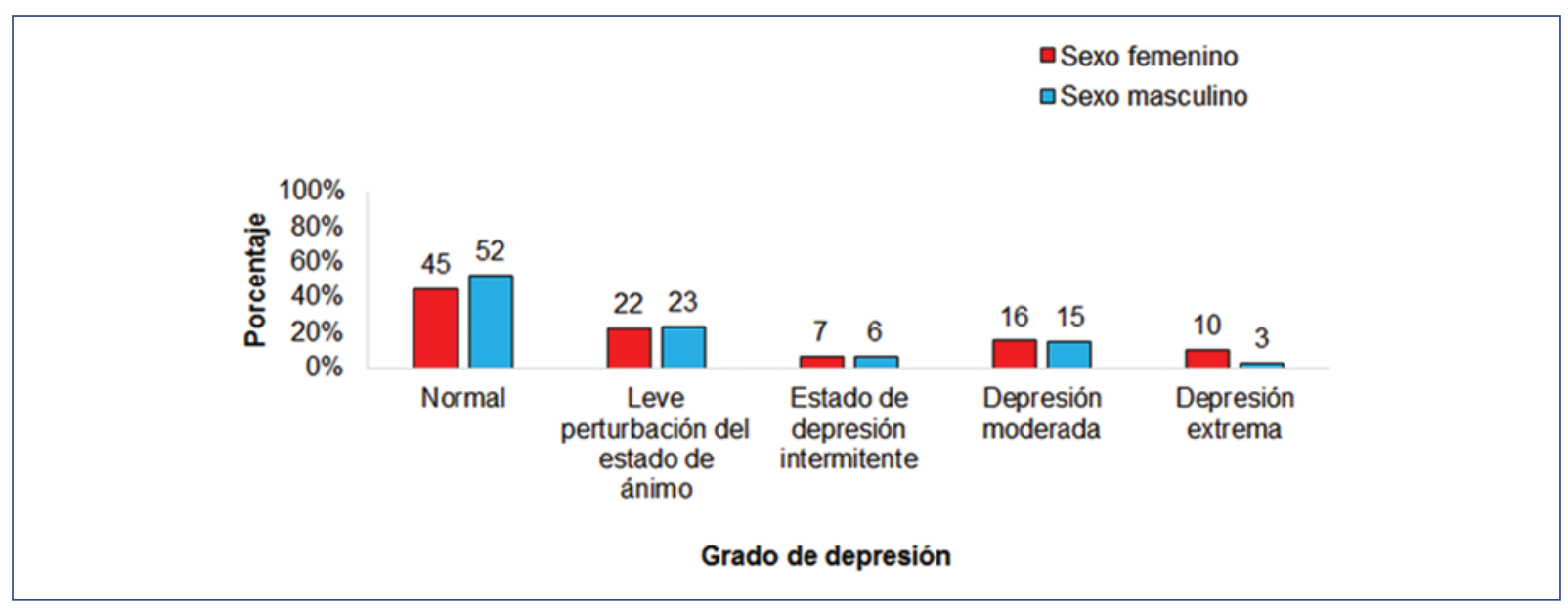

Figura 2. Porcentaje de los grados de depresión según el sexo en adolescentes de ascendencia maya.

Tabla 1. Correlaciones parciales entre disfunción temporomandibular (DTM) y depresión en adolescentes de ascendencia maya

\begin{tabular}{|l|c|c|}
\hline DTM & Correlación & $\mathbf{p}^{*}$ \\
\hline Por sexo & 0.152 & 0.028 \\
\hline Por edad & 0.169 & 0.014 \\
\hline${ }^{*}<0.05$ estadísticamente significativo. & \\
\hline
\end{tabular}

mayor estado de depresión intermitente (10\%) en comparación con los adolescentes tempranos (4\%). Por otro lado, esta relación fue inversa para la depresión extrema, ya que los adolescentes tempranos presentaron una mayor prevalencia de este grado $(9 \%)$ en comparación con los tardíos (5\%). De igual forma, se analizó si existían diferencias en el grado de depresión según el grupo de edad. No se encontraron diferencias estadísticamente significativas $(U=5317.5 ; p=0.901)$; es decir, las prevalencias de los grados de depresión resultaron similares para ambos grupos de edad.

Posteriormente se realizó un análisis similar por sexo y las prevalencias fueron relativamente similares para ambos sexos, ya que no hubo diferencias estadísticamente significativas $(U=4856 ; p=0.145)$; es decir, los niveles de depresión fueron similares para el sexo masculino y el sexo femenino (Figura 2).

Por último se realizó una prueba de correlación no paramétrica de Spearman entre los resultados de DTM y el grado de depresión. Se encontró una relación significativa, leve y directamente proporcional, entre ambas variables $(r=0.169 ; p=0.014)$; es decir, que a mayor grado de DTM, existió un mayor grado de depresión en los participantes.

También se realizaron correlaciones parciales, eliminando el efecto de las variables edad y sexo, mediante correlaciones parciales no paramétricas de Spearman (Tabla 1). Incluso eliminando el efecto del sexo y la edad, la relación entre la DTM y la depresión continuó siendo significativa y directamente proporcional.

\section{Discusión}

La DTM es un trastorno que afecta las estructuras anatómicas de la cabeza, el cuello y la boca, y está provocada por numerosos factores, entre ellos el estado de salud psicológico y mental. Por otro lado, la depresión es una enfermedad resultante de un proceso anómalo en las interacciones químicas del cerebro de quien la padece. Este trastorno incrementa su prevalencia con la edad y se caracteriza por la presencia constante de tristeza profunda, languidez y evidente deseo de soledad, acompañados por signos y síntomas neurovegetativos y motrices ${ }^{18}$.

En el presente estudio se observó que el $73.4 \%$ de los adolescentes padecen algún grado de DTM, y el sexo femenino es el más afectado. También se encontró algún tipo de depresión en el $51.9 \%$ de las adolescentes. Estos resultados difieren de los aportados por Castillo-Gamboa, et al. ${ }^{18}$ en su estudio realizado en 82 adolescentes de 11-16 años de edad en una secundaria de la zona sur de la periferia de la ciudad de Mérida, Yucatán, México, en el cual encontraron DTM en el $65.9 \%$ de los individuos. Dicha muestra también reportó 
que el $68.3 \%$ presentaron desbalances negativos en el estado de ánimo y psicológico ${ }^{18}$.

En otro estudio realizado en Ciudad de México, en 120 personas de 14 a 25 años, se encontró una prevalencia de DTM del $46.9 \%$. El sexo femenino presentó la mayor prevalencia de DTM $(52.9 \%$ vs. $37.9 \%$ en el sexo masculino). Este estudio concuerda con factores psicológicos y psicosociales de aspecto negativo, ya que el $44.6 \%$ presentó algún desbalance emocional en el sexo femenino y el $23.6 \%$ en el sexo masculino ${ }^{19}$.

La mayor prevalencia de DTM en el sexo femenino puede deberse a las características fisiológicas, en particular a las variaciones hormonales y a las estructuras en el tejido conectivo y el músculo. La mayor laxitud de estas estructuras, que se relaciona con los niveles de estrógenos, puede explicar por qué estos tejidos son menos capaces de soportar la presión funcional, lo que lleva al desarrollo de DTM con dichas características. Asimismo, los estrógenos influyen en el estado anímico, agravándolo y haciéndolo vulnerable al desarrollo progresivo de la depresión ${ }^{20,21}$.

Es importante mencionar que, dentro del amplio rango de factores que agravan la DTM, los más complicados son los de origen psicológico y emocional para el área odontológica, que debe apoyarse en otras disciplinas para poder entender esta naturaleza y, de esa manera, realizar un diagnóstico certero. Otra complicación adicional se menciona en estudios que afirman que ambas variables podrían depender una de la otra, ya que un alto nivel de duración e intensidad del dolor puede ser un factor determinante en el aumento de la depresión y la ansiedad en pacientes con dolores crónicos. Por otra parte, los sentimientos de enojo y angustia a consecuencia de esa condición generan una alta tensión y estrés en el paciente, lo que desencadena trastornos de la ATM. Un último aspecto es la posibilidad de que el desequilibrio químico en el cerebro de las personas deprimidas pueda generar un procesamiento anómalo de la sensación de dolor. Algunos autores han concluido que existe una relación de moderada a fuerte entre la depresión y la ansiedad y los síntomas de dolor de la ATM ${ }^{5,22}$.

Al finalizar esta investigación se logró sentar un precedente sobre el estado de salud psicológica y odontológica en la población adolescente de Yobaín, Yucatán, México, ya que no existen otros estudios similares que permitan realizar una comparativa. Además, dicha investigación fue dividida en las dos categorías de adolescencia descritas por la UNICEF para una mejor comprensión de los objetivos tratados. Se observó que, a mayor grado de DTM, mayor grado de depresión en los adolescentes de ascendencia maya. Se establece una pauta para acudir a otras áreas médicas, como la psiquiatría, la neurología y la neuropsiquiatría infantil, ya que, según los presentes resultados, la frecuencia de depresión leve a moderada es más alta en la adolescencia tardía; sin embargo, la mayor frecuencia de los niveles más altos de depresión se observó en la adolescencia temprana. Una explicación de ello recae en los procesos que el individuo experimenta durante la transición a la adolescencia.

Para esta investigación se siguieron los preceptos éticos para las investigaciones médicas en seres humanos de la Declaración de Helsinki de la Asociación Médica Mundial. Uno de los principios es el propósito principal de la investigación médica en seres humanos, que es comprender las causas, la evolución y los efectos de las enfermedades, y mejorar las intervenciones preventivas, diagnósticas y terapéuticas (métodos, procedimientos y tratamientos). Incluso, las mejores intervenciones probadas deben ser evaluadas continuamente a través de la investigación para que sean seguras, eficaces, efectivas, accesibles y de calidad. El presente estudio se realizó en un grupo marginado de adolescentes de ascendencia maya para determinar su estado de salud, tanto estomatognática como psicológica, para dejar precedente de ambas prácticas en estos grupos vulnerables y frágiles, tal vez poco atendidos por la sociedad.

\section{Responsabilidades éticas}

Protección de personas y animales. Los autores declaran que para esta investigación no se han realizado experimentos en seres humanos ni en animales.

Confidencialidad de los datos. Los autores declaran que han seguido los protocolos de su centro de trabajo sobre la publicación de datos de pacientes.

\section{Derecho a la privacidad y consentimiento infor-} mado. Los autores han obtenido el consentimiento informado de los pacientes y/o sujetos referidos en el artículo. Este documento obra en poder del autor de correspondencia.

\section{Conflicto de intereses}

Los autores declaran no tener ningún conflicto de intereses.

\section{Financiamiento}

Ninguno. 


\section{Bibliografía}

1. De la Torre-Rodríguez E, Aguirre-Espinosa I, Fuentes-Mendoza V, Peñón-Vivas PA, Espinosa-Quirós, Núñez-Fernández D. Factores de riesgo asociados a trastornos temporomandibulares. Rev Cubana Estomatol. 2013:50:364-73.

2. Cardenas-Erosa R, Mendiburu-Zavala C, Cortes-Carrillo D, Lugo-Ancona $P$, Navarro D, Vázquez-Paredes D. Características clínicas relacionadas con la presencia de ruidos en la articulación temporomandibular. IntraMed J. 2013;2:1-5

3. Gutiérrez J, Domínguez M, Escudero P, García J, Vicente M, Manchini T, et al. Anatomía craneofacial. Montevideo: Unidad de Publicaciones de la Facultad de Odontología, Universidad de la República; 2015.

4. Araneda P, Oyarzo J, González M, Figueroa C. Intervención psicológica en trastornos temporomandibulares: revisión narrativa. J Oral Res. 2013;2:86-90

5. Kindler S, Samietz S, Houshmand M, Grabe HJ, Bernhardt O, Biffar R, et al. Depressive and anxiety symptoms as risk factors for temporomandibular joint pain: a prospective cohort study in the general population. J Pain. 2013:13:1188-97.

6. Omnia.com.mx. En el Día Mundial de la Salud, hablemos de depresión México; 2017. Disponible en: http://www.omnia.com.mx/noticia/21790.

7. González-Forteza C, Hermosillo-de la Torre AE, Vacio-Muro M, Peralta R, Wagner FA. Depresión en adolescentes. Un problema oculto para la salud pública y la práctica clínica. Bol Med Hosp Infant Mex. 2015;72:149-55.

8. Instituto Nacional de Estadística y Geografía (INEGI). México; 2015. inegi.org.mx.

9. UNICEF. Fondo de las Naciones Unidas para la Infancia. Nueva York; 2017. Unicef.org.

10. La Jornada Maya. Balam L. Repuntan casos de suicidio entre menores en Yucatán. Yucatán; 2019. Disponible en: https://www.lajornadamaya. $\mathrm{mx} / 2019-02-18 /$ Repuntan-casos-de-suicidio-entre-menores-en-Yucatan.

11. La Jornada sin Fronteras. Records de suicidio en Yucatán: 235 en 2018. Ciudad de México; 2019. Disponible en: https://www.jornada.com.mx 2019/01/03 , estados.
12. Diario de Yucatán. Noh-Estrada A. ¿Por qué tanto suicidio en Yucatán? Yucatán; 2019. Disponible en: http://yucatan.com.mx/merida/por-que-tanto-suicidio-en-yucatan.

13. Rani S, Pawah S, Gola S, Bakshi M. Analysis of Helkimo index for temporomandibular disorder diagnosis in the dental students of Faridabad city: a cross-sectional study. J Indian Prosthodont Soc. 2017; 17:48-52.

14. Da-Cunha SC, Nogueira RV, Duarte AP Vasconcelos BC, Almeida-R de A Analysis of Helkimo and Craniomandibular indexes for temporomandibular disorder diagnosis on rheumatoid arthritis patients. Braz $\mathrm{J}$ Otorhinolaryngol. 2007;73:19-26

15. Norma Oficial Mexicana NOM-013-SSA2-2015, para la prevención y control de enfermedades bucales. Diario Oficial de la Federación: SEGOB/Secretaría de Salud; 2016.

16. Beck AT, Weissman A, Lester D, Trexler $L$. The measurement of pessimism: the hopelessness scale. Consult J Clin Psychol. 1974,42:861-5.

17. Castro M. Bioestadística aplicada en investigación clínica: conceptos básicos. Rev Med Clin Las Condes. 2019;30:50-65.

18. Castillo-Gamboa GA, Medina-Fernández JA, Medina-Fernández IA, Candila-Celis JA, Gazca-Vázquez SE. Trastorno temporomandibular relacionado con ansiedad en adolescentes de una zona suburbana de Yucatán, México. Ciencia Humanismo Salud. 2018;5:2-8.

19. Casanova-Rosado JF, Medina-Solís CE, Vallejo-Sánchez AA, Casanova-Rosado AJ, Hernández-Prado B, Ávila-Burgos L. Prevalence and associated factors for temporomandibular disorders in a group of Mexican adolescents and youth adults. Clin Oral Invest. 2006;10:42-9

20. Minghelli B, Morgado M, Caro T. Association of temporomandibular disorder symptoms with anxiety and depression in Portuguese college students. J Oral Sci. 2014;56:127-33.

21. Okeson JP, de Leeuw R. Differential diagnosis of temporomandibular disorders and other orofacial pain disorders. Dent Clin North Am. 2011; 55:105-20.

22. Hernández-Romero Y, Tápanes-Domínguez A, Casanova-Díaz C, Cárdenas-Peña L, Saavedra de la Cruz A. Niveles de ansiedad y depresión en un grupo de pacientes con trastornos en la articulación témporo-mandibular. Rev Hosp Psiquiátrico de la Habana. 2013;1:1-5. 